\title{
Les dysfonctions érectiles chez le diabétique : Rôle des facteurs neurologiques
}

\author{
1 S. M.GUEYE, 2 S. N. DIOP, 1 P. A. FALL, 1 E.K. DAGADOU, 1 M. O. C. ABDALLAHI, \\ 1 M. BA, A. MENSAH 1 \\ 1 Service d'Urologie, Hôpital Aristide Le DANTEC \\ 2 Centre anti-diabétique M. Sankalé , Hôpital Abass NDAO DAKAR - SENEGAL
}

RESUME

But : L'objectif de ce travail était d'évaluer les facteurs étiologiques de la dysfonction érectile chez le diabétique.

Matériel et Méthodes : Il s'agit d'une étude prospective ayant porté sur 69 patients diabétiques souffrant d'une dysfonction érectile. Nous avons étudié des variables ayant trait à l'identité, le statut marital et les antécédents médico-chirurgicaux notamment le diabète et les maladies vasculaires, neurologiques, endocriniens, et urologiques. Nous avons aussi recherché la notion d'intoxication alcoolique et tabagique et la prise de médicaments anti-diabétiques ou autres. Chaque patient a fait l'objet d'un examen clinique complet. Les complications dégénératives du diabète ont été recherchées et notées le cas échéant. L'analyse statistique s'est faite selon la méthode des cas-témoins. Le groupe des diabétiques a été comparé à un lot témoin de 138 patients diabétiques mais n'ayant pas de dysfonctionnement érectile.

Résultats : Les complications du diabète notamment neurologiques étaient bien corrélées au risque d'insuffisance érectile ( $p=$ 0,0004). De même, la prévalence de la dysfonction érectile augmentait avec la durée d'évolution du diabète $(p=0,049)$.

Conclusion : Si l'étiologie de la dysfonction érectile chez le diabétique est multi-factorielle, les complications dégénératives en particulier neurologiques semblent jouer un rôle prépondérant.

Mots clés : Dysfonction érectile, diabète, neuropathie

\section{INTRODUCTION}

Les dysfonctions érectiles compliquent souvent l'évolution du diabète sucré. La prévalence pouvant atteindre 25 à $60 \%$ selon les séries [2, 3, 6, 9] Les mécanismes physiopathologiques restent controversés. En effet, des perturbations psychologiques sont toujours présentes même en cas d'atteinte organique de type vasculaire, neurologique ou autres.

L'objectif de ce travail était d'évaluer les facteurs étiologiques de la dysfonction érectile chez le diabétique.

\section{PATIENTS ET MÉTHODES}

Ce travail prospectif a porté sur une série de 69 diabétiques souffrant d'une dysfonction érectile parmi un groupe consécutif de 431 hommes diabétiques suivis au centre anti-diabétique sur une période de 9 mois. Le lot témoin était constitué de 138 diabétiques choisis parmi les 362 patients ne présentant pas de trouble érectile. Tous les patients ont fait l'objet d'un examen clinique complet qui a permis de recueillir des paramètres liés à l'identité : âge, ethnie, origine géographique, niveau de scolarisation, situation matrimoniale, nombre d'épouses, et nombre d'enfants. Les antécédents médicaux et chirurgicaux notamment vasculaires, neurologiques, endocriniens et urologiques ont été notés. La notion d'intoxication alcoolo-tabagique et la prise de médicaments ont été relevées. L'évaluation de

Correspondance : S. M. GUEYE, BP 6039, Dakar Etoile, Sénégal. E-mail : smgueye@metissacana.sn 
l'équilibre du diabète a été faite sur la mesure des 4 derniers taux de la glycémie.

La saisie et l'exploitation des données a été faite avec le logiciel Epi info version 6 . L'analyse statistique a été faite selon la méthode des cas-témoins. Pour la comparaison des deux groupes, nous avons utilisé le test de chi 2 pour les variables qualitatives et le test de Mann-Whitney pour les variables quantitatives. Le seuil de signification a été fixé pour $\mathrm{p}$ $<0,05$.

\section{RÉSULTATS}

L'âge moyen des diabétiques présentant une dysfonction érectile était de 54 ans avec des extrêmes de 27 et 81 et un écart-type de 10 . Celui du groupe témoin était de 53 ans avec des extrêmes de 28 et 80 ans.

L'âge moyen des malades au moment du dépistage du diabète était de 48,52 ans avec des extrêmes de 17 et 74 ans et un écart-type de 10,03. Dans le groupe témoin, l'âge moyen au moment du dépistage du diabète était de 49,02 ans avec des extrêmes de 25 et 71 ans et un écart-type de 9,55. La durée moyenne d'évolution du diabète était de 6 ans. Cette durée était inférieure à 10 ans dans $80,7 \%$ des cas. Il s'agissait d'un diabète non insulino-dépendant (DNID) dans 90,8 \% des cas et d'un diabète insulino-dépendant (DID) dans 9,2\% des cas. Le diabète était équilibré chez $61,4 \%$ des malades et $60,9 \%$ des témoins. Le mode d'installation des troubles érectiles était progressif dans $88,5 \%$ des cas, brutal dans $4,3 \%$ des cas. La dysfonction érectile était intermittente dans $7,2 \%$ des cas. La persistance d'une érection matinale était notée par $42 \%$ des malades. La rigidité de l'érection était minime dans $42 \%$ des cas, modérée dans $49,3 \%$ des cas et nulle chez $8,7 \%$ des patients. Une complication dégénérative a été retrouvée dans $68,12 \%$ des cas. Dans $57,5 \%$ des cas, il s'agissait d'une complication neurologique. Les autres facteurs étiologiques retrouvés étaient un tabagisme dans $37,2 \%$ des cas, un alcoolisme dans $6,3 \%$, et une hypertension artérielle dans 19,9\% des cas. Tous les patients étaient soumis à un régime hypoglucidique. Un sulfamide hypoglycémiant a été associé dans $31,4 \%$ des cas.
La comparaison des deux groupes a permis d'évaluer l'influence des facteurs étiologiques sur la survenue de la dysfonction érectile. En utilisant le test de Mann-Whitney pour les variables quantitatives comme l'âge et la durée d'évolution du diabète, les valeurs respectives de $\mathrm{p}$ étaient de 0,985 et 0,126 . Le test de chi 2 a été utilisé pour les variables qualitatives qui ont fait l'objet de comparaison telles que le type de diabète, les complications dégénératives, les types de traitement du diabète, l'hypertension artérielle et le tabagisme. Il ressort de notre analyse que la durée d'évolution du diabète $(p=0,049)$ ainsi que les complications dégénératives $(\mathrm{p}=0.00043)$ étaient les facteurs statistiquement liés au risque de survenue d'une dysfonction érectile chez le diabétique. Parmi ces complications, les neuropathies diabétiques étaient les plus significatives ( $\mathrm{p}=$ $0,0004)$. L'hypertension artérielle $(p=0,464)$, le traitement du diabète $(p=0,980)$ et le tabagisme ( $p=0,477)$ ne semblaient pas jouer un rôle dans le risque de survenue d'une dysfonction érectile.

\section{DISCUSSIONS}

L'âge est apparu comme un facteur de confusion, mais ne semble pas intervenir de façon significative dans la survenue des troubles érectiles chez nos patients diabétiques $(p=$ 0,948). Cependant, si ce constat confirme certains auteurs [4], d'autres pensent que les troubles érectiles sont bien âge-dépendants [6, 7]. Il est apparu un lien entre la durée d'évolution du diabète et la survenue de la dysfonction érectile $(p=0,049)$. Ce résultat corrobore Fossati et al [7] qui pensent que la fréquence des troubles érectiles augmente avec l'ancienneté du diabète, pouvant dépasser $50 \%$ après 10 ans d'évolution. En revanche le type de diabète ne semble pas jouer un rôle dans la survenue des troubles érectiles $(p=0,865)$. Il se pourrait que le faible nombre de diabétiques insulino-dépendants de même que leur jeune âge puisse constituer un biais dans notre analyse.

Les complications dégénératives semblent jouer un rôle non négligeable dans la survenue de la dysfonction érectile $[1,5,9,11,12]$. Une neuropathie diabétique, a été retrouvée chez $62,32 \%$ des patients souffrant d'une insuffi- 
sance érectile et chez $55,1 \%$ des sujets témoins. La différence entre les deux groupes apparaît significative $(p=0,0004)$. Buvat et al [2], Bemelmans et al [1] et Ellenberg [5] notent que la neuropathie périphérique représente une cause majeure des troubles érectiles chez le diabétique. Les données de notre étude ne nous ont pas permis de discuter le rôle des complications de type microangiopathie comme l'altération tissulaire des corps caverneux et de type macroangiopathie telle que l'athérome. Cependant, leur relation avec la survenue des troubles érectiles chez le diabétique a été rapportée par d'autres auteurs [10, 13]. Nous n'avons pas noté d'influence de la nature du traitement du diabète sur la fréquence des troubles érectiles $(p=0,980)$. Cependant, Kolodny et al [8] pensent qu'il existe une plus faible fréquence des troubles sexuels chez le diabétique non insulino-dépendant (DNID) traités par le régime seul sans qu'aucune explication claire puisse être donnée à ce fait. Les facteurs psychiques interviennent de façon notable dans la survenue des troubles érectiles. Nous avons trouvé une fréquence légèrement plus importante du stress et de l'anxiété dans le groupe de diabétiques présentant une dysfonction érectile sans pour autant en tirer une relation statistiquement significative.

L'étiologie des troubles de l'érection chez le diabétique est multifactorielle. Nos résultats suggèrent que ce sont surtout les complications dégénératives et principalement la neuropathie qui sont délétères sur la fonction sexuelle.

\section{RÉFÉRENCES}

1. BEMMELMANS B. L., MEULEMANS E. , DOESBURG N. S, BEBRUYNE F. Erectile dysfunction in diabetic men: the neurological factor revisited $\mathrm{J}$. Urol., 1994, 151, 884-889

2. BUVAT J., LEMAIRE A., BUVAT-HERBAUT M., GUIEU J.D., BAILLEUL J. P., FOSSATI P. Comparative investigations in 26 impotent and 26 non-impotent diabetic patients. J. Urol., 1984, 133, 34-39

3. BUVAT J., LEMAIRE A. L'impuissance diabétique a le plus souvent une origine plurifactorielle. Rev. Fr. Endocrinol. clin. 1985, 26, 2

4. DIOKNO A. C., BROWN M. B., HERZOG A. R. Sexual function in the elderly. Arch. Intern. Med.,1990, 150
5. ELLENBERG M. Impotence and diabetes: the neurologic factor. Ann. Intern. Med., 1971,75,213-219

6. FELDMAN H.A, GOLDSTEIN I, HATZICHRISTOU D. G, KRANE R.J, McKINLAY J. Impotence and its medical and psychosocial correlates: results of the Massachusetts Male Aging Study. J. Urol., 1994, 151, 54-61

7. FOSSATI P., BUVAT J., LEMAIRE A. Fonctions sexuelles et de reproduction chez les diabétiques Journée diabétologie Hôtel-Dieu. Paris: Flammarion, 1984, 269-286

8. KOLODNY R. C., GOLDSTEIN H. H., BARNETT D. N., KAHN B. Sexual dysfunction in diabetic men. Diabetes, 1974, 23, 306-309

9. McCULLOCH D. K., YOUNG R. G., PRESCOTT RJ., CAMPBELL I., W., CLARKE B. F. The natural history of impotence in diabetic men. Diabetologia, 1984, $26 / 6,437-440$.

10. ROSEN M. P, GREENFIELD A. J., WALKER T. G., GRANT P., DUBROW J., BETTMANN M. A, FRIED L. E, GOLDSTEIN I. Cigarette smoking: an independent risk factor for atherosclerosis in the hypogastriccavernous arterial bed of men with arteriogenic impotence. J. Urol., 1991, 145, 759-763.

11. SCHERB W. H. Neurophysiological evaluation of erectile dysfunction. In U. Jonas, W. F. Thon, C. G. Stief (Eds). Erectile dysfunction Springer-Verlag, 1991, 178-186

12. VALENSI $\mathrm{P}$ La neuropathie autonome diabétique. Presse Méd., 1993, 22, 4, 166-172

13. WERYHA G , LECLERE J Diabète et sexualité masculine: données épidémiologiques Prog. Androl., 1989, $2,117-126$.

\section{ABSTRACT}

\section{Erectile dysfunction in the diabetics : Role of neurological factors.}

\author{
S. M. GUEYE, S. N. DIOP, P. A. FALL, \\ E. K. DAGADOU, M. O. C ABDALLAHI, \\ M. BA, A. MENSAH
}

Objective : To assess the etiological factors of erectile dysfunction in male diabetics.

Material and methods : We have performed a prospective evaluation including 69 diabetic patients suffering from erectile dysfunction. Studied parameters including age, type and duration of diabetes, complications, treatments and associated risk factors were analysed. Comparison was done with a control 
group of 138 diabetic patients without erectile dysfunction.

Results : There was a significant difference between the diabetic with neurologic complications and the others without neuropathy ( $p$ $=0.0004$ ). The duration of the diabete was was another risk factor of erectile dysfunction ( $p$ = 0.049)

Conclusion: We confirm various authors who demonstrated that diabetic impotence seems to be mainly neuropathic in etiology even though it was a multifactorial discomfort.

Key words : Erectile dysfunction, diabetes, neuropathy 